Konrad-Zuse-Zentrum für Informationstechnik Berlin

\title{
Fare Planning for Public Transport
}




\title{
Fare Planning for Public Transport*
}

\author{
Ralf Borndörfer Marika Neumann Marc E. Pfetsch
}

\begin{abstract}
In this paper we introduce the fare planning problem for public transport which consists in designing a system of fares maximizing revenue. We propose a new simple general model for this problem. It is based on a demand function and constraints for the different fares. The constraints define the structure of the fare system, e.g., distance dependent fares or zone fares. We discuss a simple example with a quadratic demand function and distance dependent fares. Then we introduce a more realistic discrete choice model in which passengers choose between different alternatives depending on the number of trips per month. We demonstrate the examples by computational experiments.
\end{abstract}

\section{Introduction}

In public transport, fares are among the most important factors that influence the number of passengers and with it the revenue. Especially in local public transport, there are several ticket fares that can be individually adjusted, e.g., fares for single tickets, monthly tickets, distance dependent tickets, etc. From the viewpoint of a public transport company or transit authority it would therefore be interesting to optimize the system of fares such that its revenue is maximized. Since public transport companies often work at deficit, the objective is then to reduce the deficit as much as possible.

Of course, in many countries the problem to design fares is inherently political, limiting the degree of freedom in planning. In reality, fares are often more the outcome of negotiations than really planned. Nevertheless, it would be useful to provide the planner with mathematical programming models that support his or her decision. This paper is meant as a first step in this direction.

We propose to consider the fare planning problem (FPP) in public transport which consists in designing a fare system such that revenue is maximized. A fare system consists of several prices for different types of tickets.

\footnotetext{
*Supported by the DFG Research Center MatheON "Mathematics for key technologies" Address of the authors: Konrad-Zuse-Zentrum für Informationstechnik Berlin, Takustr. 7, 14195 Berlin, Germany; Email: \{borndoerfer, marika.neumann, pfetsch\}@zib.de
} 
Given a fare system, the revenue is given by the fares times the number of passengers using each ticket type in this situation. Our fundamental assumption is that the number of passengers in dependence on the fare can be expressed by a demand function. This requires that passengers act rationally and have full knowledge of the all alternatives. In this situation the demand function will be non-increasing: the higher the fare, the lower the number of passengers willing to use public transport. We note, however, that it it is hard to estimate a demand function which describes reality with acceptable quality; see the discussion in Section 2.

The fare planning problem and related problems have received little attention in the operations research literature up to now. To our knowledge there is no publication considering a mathematical optimization model for this problem. On the other hand, demand functions are well established in economics and the public transport literature [11]. Furthermore, the geometric or structural design of fare zone systems has been treated by Hamacher and Schöbel [7, 8].

In this paper, we propose a simple general mathematical programming model for FPP, see Section 3. The model allows for a general coupling of individual fares of passengers to create a complete fare system.

In a first "textbook" example in Section 5, we use a simple quadratic demand function and only one ticket type. The fare consists of a part proportional to the traveled distance plus a basic fare. Similar systems are typically used in public rail transport. Such models may also become more interesting for local public transport systems in the future, once electronic tickets will be available. To illustrate our approach towards FPP, we then optimize the distance dependent fare and basic fare in order to maximize revenue and provide computational experiments.

In Section 6 we propose the usage of more realistic demand functions based on a discrete choice model. We introduce a particular model, where passengers can choose between three different travel alternatives depending on the number of trips within a given time horizon. The alternatives are a standard ticket, a reduced ticket for which a basic fare has to be paid once, and car travel. With this model we then optimize fares for the public transport alternatives and provide computational experiments.

The computational experiments are performed on data for the intercity network of the Netherlands. The data are relatively far from being realistic and hence no conclusions should be drawn for the real situation. The results are, however, meant to provide evidence for the applicability of our model to the real world situation. See Section 4 for a discussion.

The rest of this paper is organized as follows. In Section 2 we discuss properties of demand functions. Section 3 introduces the general model. The data and methods used for the computational experiments are discussed in Section 4. In Section 5 we discuss the first example. Section 6 deals with the discrete choice model. 


\section{Demand Functions}

A demand function measures the amount of clients which choose a particular option depending on input parameters. An example is the number of customers buying a product depending on its price and the prices of competing products. In this paper, we are concerned with the number of passengers which use public transport depending on the fare and possibly on the cost of other options like cars, bikes, etc.

It is not at all clear that demand functions are adequate for modeling real-world problems, i.e., that demand function can give a good enough approximation of the behavior of clients for such problems. However, demand functions are commonly used in the economic literature and in publications on planning in public transport. The reason is that otherwise a mathematical treatment of planning problems is hard, if not impossible. Following this argument, our approach towards FPP is also via demand functions. In fact, we work with concrete demand functions which measure the amount of passengers using public transport depending on the fare.

Note that the existence of such a demand function implies that passengers act rationally with respect to the change of fares. It has been observed that such an assumption hardly holds in the real world, see e.g. the discussion of Walther [14]. For instance, the behavior of passengers is not symmetric: There is a difference between increasing and decreasing fares. If the fare is increased and then decreased to the original value, the numbers of passengers using public transport probably is different from the initial values. Furthermore, after changing the fare there is a time of adaption until the behavior of passengers has stabilized. One can argue that after a long enough time the situation comes close to being symmetric ${ }^{1}$. But then this effect is covered by other changes in the system, e.g., a change in the timetable, line system, or network. This also shows that in fact an exact demand function cannot empirically be determined.

That estimating real-world demand functions is complicated is shown by the fact that experiments with a zero fare policy do not give a clear picture of the passenger's behavior, see, e.g., Baum [1] and Storchmann [12]. In some towns the increased passenger numbers are mainly due to influenced traffic and passengers that used a bike or went by foot before.

Let us outline the class of demand functions suitable for our approach. We assume that the fare and the corresponding function values take continuous values. This is a simplification, since the number of passengers are integer and usually fares have integer values, too (when measured in correct units). We assume, however, that the number of passengers is large enough and fares can be rounded; hence, these effects are negligible. Since passengers usually base their decisions on discrete conditions, e.g., whether there is

\footnotetext{
${ }^{1}$ Note that purchasing a car is a long-term decision, so the time could be quite long.
} 
a car or a parking place available, one cannot assume that the demand function itself is continuous. In our two examples below, however, the demand functions will even be differentiable. This enables an easy optimization of the corresponding mathematical programming models.

As noted above, it follows from our assumption that passengers act rationally that the demand function will be non-increasing. Furthermore, the demand function and fares have to be nonnegative. Summarizing, our demand functions take the following form:

$$
d: \mathbb{R}_{+}^{n} \rightarrow \mathbb{R}_{+},
$$

when there are $n$ different fares. Furthermore, $d$ is (componentwise) nonincreasing. The revenue $r: \mathbb{R}_{+}^{n} \rightarrow \mathbb{R}_{+}$for this demand type is then the inner product $r(p)=p^{\mathrm{T}} d(p)$. See Figure 1 of Section 5 for examples. In practice, the fares should only vary in a small interval around the current fare, such that the function gives a reliable estimate of the behavior of the passengers, but is not too small to be useless.

Note that a fare of 0 is likely to be a source of discontinuity and one has to be careful near this value. Nevertheless, in our first example we allow fares in the range of $[0, \infty)$. In fact, in this example we make assumptions about the number of passengers traveling at a zero fare to calibrate our demand function. The point 0 is chosen for convenience only, and the evaluations of the resulting demand function are far from this point.

In the literature there are basically two types of demand functions. One is the function one gets from a fixed fare elasticity $\epsilon$, which is defined as the relative demand change in percent divided by the relative fare change in percent. For the case of one fare and fixed elasticity, Cerwenka [5] showed that the demand function is

$$
d(p)=c \cdot p^{\epsilon}
$$

for some constant $c \in \mathbb{R}$. In the literature one often uses values around $\epsilon=-0.3$; this value is usually attributed to Curtin and Simpson [6]. As long as $\epsilon<0$ the above demand function is undefined at zero and hence not applicable for experiments where the fare is reduced to zero. We do not use elasticities in this paper.

The other type of demand function used in the literature arises from so called discrete choice analysis. To get an approximation of the demand function, one uses questionnaires on the planned behavior of passengers ("stated preferences") in addition to measured behavior ("revealed preferences"), see for instance Vrtic and Axhausen [13]. Such data can then be used to design a discrete choice model. Such models include a certain random effect on the preferences of passengers which takes the inability to measure the real preferences into account. This also forces the resulting demand function to be continuous. See Section 6 for more information. 


\section{A General Model}

For our general model for optimizing the revenue, we are given a public transport network $N$ with $k$ different ticket types; for instance, a ticket can be for a single trip, for a day, a month etc. Additionally, we are given a set $D$ of (ordered) pairs of nodes, so called origin-destination pairs (OD-pairs). For each OD-pair $(s, t) \in D$ and each ticket type $i$, we have fares $p_{s t}^{i}$ for traveling from $s$ to $t$ with ticket type $i$. These fares are in general not independent from each other, see below. Furthermore, we have bounds $a_{s t}^{i} \leq p_{s t}^{i} \leq b_{s t}^{i}$ for each fare, where $a_{s t}^{i} \geq 0$ and $b_{s t}^{i}$ may be infinity. We write $\boldsymbol{p}_{s t}=\left(p_{s t}^{1}, \ldots, p_{s t}^{k}\right)$ for the vector of all fares from $s$ to $t$ and $\boldsymbol{p}=\left(\boldsymbol{p}_{s t}\right)$ for the vector of all fares. Analogous notation is used for $a_{s t}^{i}$ and $b_{s t}^{i}$.

Similarly,

$$
d_{s t}^{i}:\{\boldsymbol{p}: \boldsymbol{a} \leq \boldsymbol{p} \leq \boldsymbol{b}\} \rightarrow \mathbb{R}_{+},
$$

gives the amount of passengers that travel through $N$ with ticket $i$ from $s$ to $t$ depending on all fares $\boldsymbol{p}$. The total revenue for $(s, t) \in D$ and ticket type $i$ is computed as

$$
r_{s t}^{i}\left(\boldsymbol{p}_{s t}\right):=p_{s t}^{i} \cdot d_{s t}^{i}\left(\boldsymbol{p}_{s t}\right) \quad \text { and } \quad r_{s t}\left(\boldsymbol{p}_{s t}\right):=\sum_{i=1}^{k} r_{s t}^{i}\left(\boldsymbol{p}_{s t}\right)
$$

is the total revenue for all travels from $s$ to $t$.

The goal of the fare planning problem is to design the fares $p_{s t}^{i}$ such that the revenue is maximized. Thereby several constraints can be imposed on the fares, e.g.:

- $p_{s t}=p_{t s}$ for all $(s, t) \in D$ (symmetric fares).

- $p_{s t}^{i}=p_{u v}^{i}$ for all $(s, t) \in D,(u, v) \in D$ (e.g. a unit fare ticket).

- $p_{s t}^{i}=$ fare proportional to the distance between $s$ and $t$.

- $p_{s t}^{i}=c \in \mathbb{R}$, if the distance between $s$ and $t$ is below a certain bound (short travel ticket).

Such constraints are usually needed to improve the comprehensibility of the fare system or to enforce certain political objectives.

Our general model can therefore be written as:

$$
\begin{array}{cc}
\max & \sum_{(s, t) \in D} r_{s t}\left(\boldsymbol{p}_{s t}\right) \\
\text { s.t. } & \boldsymbol{p} \in P \\
& \boldsymbol{a} \leq \boldsymbol{p} \leq \boldsymbol{b},
\end{array}
$$

where $P \subseteq \mathbb{R}^{n}, n:=k \cdot|D|$. The set $P$ captures the constraints on the fares. In fact, all of the constraints listed above are linear in the fares. Therefore in this paper, $P$ is always a polyhedron, but we do not make explicit use of this. 
When no constraints are given on the fares, i.e., $P=\mathbb{R}^{n}$, and the demand function is differentiable, the problem amounts to find a fixed point for $d(p) / d^{\prime}(p)$. In these cases this can be done analytically in a straightforward way.

In general, however, the above model is a quite hard nonlinear programming problem. For instance, note that the revenue is usually not convex, see Figure 1 for examples, and might even be discontinuous. Nevertheless, in our computational experiments we could solve the resulting programs by standard methods with reasonable quality.

\section{Data for Computational Experiments}

In the following two sections we provide computational experiments with our approach on particular examples.

Obtaining data for such experiments is quite hard. There are almost no publicly available OD-data which capture traffic in public transport. Even less data is available which covers all kind of traffic, i.e., all types of public transport and cars. Moreover, corresponding fares, traveling times, and distances are hard to get. Although there are lots of publications dealing with discrete choice models, only few deal with several different user groups or destinations. Therefore, the computational experiments presented in this paper should be treated with care and are meant to provide evidence of the practicability of our approach only.

Our experiments were conducted on data for the intercity network of the Netherlands and a corresponding origin-destination matrix. The data can be found in a GAMS model set up by Bussieck [3] (see also [4]). The origindestination matrix is used to calibrate our models and gives the number of passengers that want to travel between every pair of cities connected by the intercity network. The matrix is symmetric and has 420 nonzero entries. The network has 23 nodes (cities).

Furthermore, we collected the currently valid fares, distances, and travel times in the Netherlands for every pair of towns from the Internet, see Section 6.4. Note that the origin-destination data and the current fares were collected in different years and might not fit together. We think, however, that the deviation is reasonable for our experiments. With these data, the current total demand is 91791 and the current total revenue is $860991.3 €$.

All computations were performed with GAMS 2.50 and Matlab 7.

\section{A Textbook Example}

In this section we want to demonstrate the applicability of our model on a simple example. We use one ticket type with a quadratic demand function 

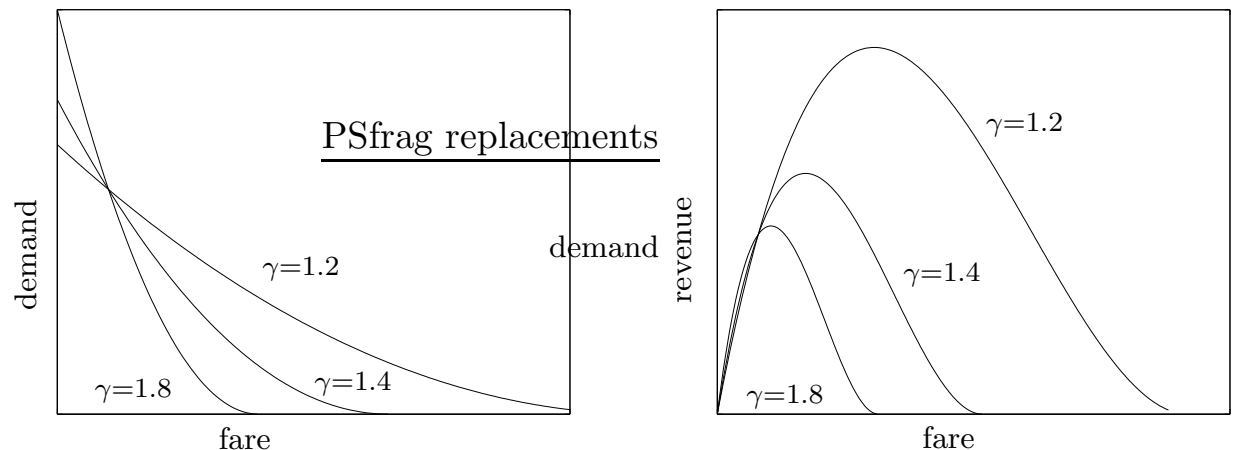

Figure 1: Left: Sketch of quadratic demand function (2) for different values of $\gamma$. Right: Resulting revenue functions.

and compute distance dependent fares. More precisely, we consider a distance fare $p_{d}$ which gives the fare per traveled distance. Additionally, we have a basic fare $p_{b}$ which has to be paid whenever public transport is used. Hence, the fare between $s$ and $t$ is

$$
p_{s t}\left(p_{b}, p_{d}\right)=p_{b}+p_{d} \cdot \ell_{s t},
$$

where $\ell_{s t}$ is the shortest distance between $s$ and $t$.

We use the following quadratic demand function

$$
d_{s t}\left(p_{b}, p_{d}\right)= \begin{cases}\alpha_{s t} \cdot\left(p_{s t}\left(p_{b}, p_{d}\right)-\beta_{s t}\right)^{2} & p_{s t}\left(p_{b}, p_{d}\right) \leq \beta_{s t} \\ 0 & \text { otherwise }\end{cases}
$$

where $\alpha_{s t}, \beta_{s t}$ are positive parameters. We also experimented with the following demand function:

$$
d_{s t}\left(p_{b}, p_{d}\right)=\alpha_{s t} \cdot e^{-\beta_{s t} \cdot p_{s t}\left(p_{b}, p_{d}\right)}
$$

(again $\alpha_{s t}, \beta_{s t}>0$ are parameters). The results are quite similar and we therefore do not present them here.

The model (1) specialized for our fare system now reads:

$$
\begin{array}{cl}
\max & \sum_{(s, t) \in D} p_{s t}\left(p_{b}, p_{d}\right) \cdot d_{s t}\left(p_{b}, p_{d}\right) \\
\text { s.t. } & p_{s t}\left(p_{b}, p_{d}\right)=p_{b}+p_{d} \cdot \ell_{s t} \\
& p_{b}, p_{d} \geq 0 .
\end{array}
$$

We fix the parameters $\alpha_{s t}$ and $\beta_{s t}$ such that the demand function interpolates between two points. As the first point, we take the current fare $p_{s t}^{0}$ with current demand $d_{s t}^{0}$, i.e., $d_{s t}\left(p_{s t}^{0}\right)=d_{s t}^{0}$ for all $(s, t) \in D$, i.e., the model is calibrated with the current situation. 

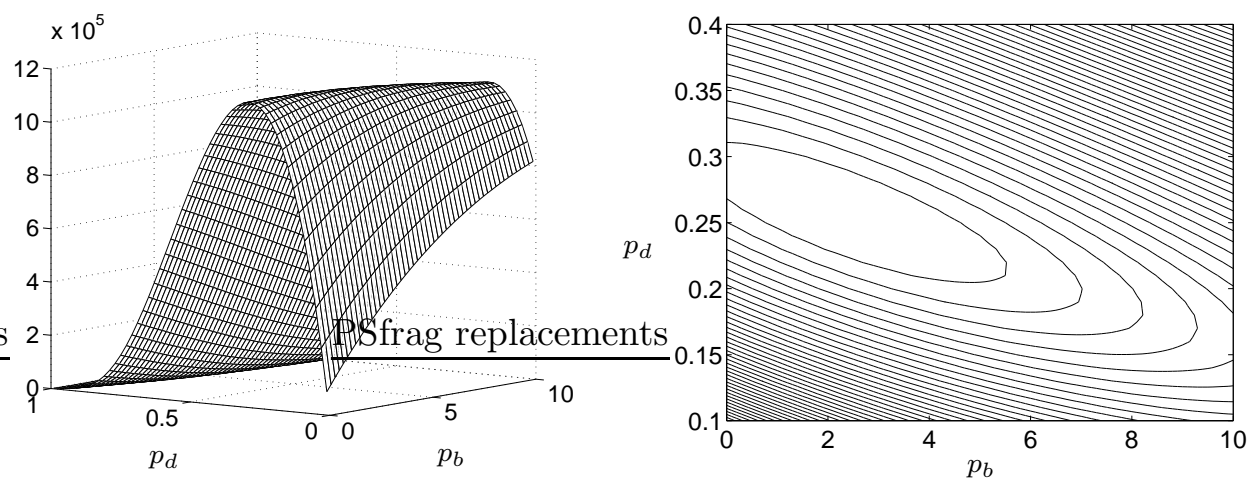

Figure 2: Textbook example. Left: Total revenue. Right: A contour plot of the total revenue. The optimum is at $p_{b} \approx 2.29$ and $p_{d} \approx 0.26$.

Table 1: Computed fares versus currently valid fares for selected trips for $\gamma=1.4$. The current fares are $p^{0}$, the current demand is $d^{0}$, while the new ones are $p$ and $d ; \ell$ denotes the distance between the selected endpoints of the travel.

\begin{tabular}{rrrrcrrrr}
\hline & & & \multicolumn{4}{c}{$p_{b}=0$} & \multicolumn{2}{c}{$p_{d}=0$} \\
$\ell$ & $d^{0}$ & $p^{0}$ & \multicolumn{1}{c}{$d$} & \multicolumn{1}{c}{$p$} & \multicolumn{1}{c}{$d$} & \multicolumn{1}{c}{$p$} & \multicolumn{1}{c}{$d$} & $p$ \\
\hline 11 & 1456 & 2.1 & 789.2 & 5.12 & 1194.8 & 3.17 & 74.0 & 10.98 \\
35 & 5826 & 5.2 & 3591.6 & 11.30 & 3983.2 & 10.11 & 3695.1 & 10.98 \\
50 & 1829 & 7.1 & 1147.6 & 15.16 & 1201.1 & 14.45 & 1481.2 & 10.98 \\
106 & 720 & 14.0 & 456.6 & 29.57 & 440.7 & 30.63 & 778.1 & 10.98 \\
150 & 24 & 19.2 & 15.1 & 40.90 & 14.2 & 43.35 & 27.9 & 10.98 \\
202 & 77 & 24.1 & 45.7 & 54.29 & 42.1 & 58.37 & 93.1 & 10.98 \\
250 & 1 & 28.5 & 0.6 & 66.64 & 0.5 & 72.24 & 1.2 & 10.98 \\
303 & 14 & 33.3 & 7.7 & 80.29 & 6.9 & 87.55 & 17.6 & 10.98 \\
\hline
\end{tabular}

As the second point we take a zero fare with demand $\gamma \cdot d_{0}$, where $\gamma$ is a parameter, i.e., $d_{s t}(0)=\gamma \cdot d_{s t}^{0}$. See Figure 1 for examples of demand functions arising from different values of $\gamma$ : The higher $\gamma$, the more sensitive passengers react to a change of the fares, i.e., the less passengers would travel with an increased fare. Similarly, the lower $\gamma$, the less sensitive passengers react to a change of the fares. When computing the fare elasticity (see Section 2) at the current fare and $\gamma=1.4$, we get $\varepsilon=-0.37$.

We performed computational experiments with this model for the intercity network of the Netherlands as described in Section 4. Figure 2 shows the resulting total revenue for $\gamma=1.4$, i.e., a passenger increase of $40 \%$ at zero fare. The optimal fares are $p_{b} \approx 2.29$ and $p_{d} \approx 0.26$. The computations were performed by standard methods without difficulties. In this case we used GAMS with Minos 5.5.

Table 1 shows a comparison between the currently valid fares and demands versus the optimal ones. Furthermore, the table shows optimal solu- 
Table 2: Results of the optimization for different values for $\gamma ; d$ denotes the total demand, $r$ the total revenue. The bold numbers indicate values that are closest to the current values, i.e., indicate values of $\gamma$ for which current fares and demand would be optimal.

\begin{tabular}{lllrr}
\hline$\gamma$ & $p_{b}$ & $p_{d}$ & $d$ & $r$ \\
\hline 1.2 & 4.08 & 0.46 & 48925 & 1755148 \\
1.4 & 2.29 & 0.26 & 57079 & 1152190 \\
1.6 & 1.7 & 0.19 & 65233 & 973588 \\
1.7 & 1.52 & 0.17 & 69311 & 929658 \\
1.8 & 1.39 & 0.16 & 73388 & 900812 \\
1.9 & 1.29 & 0.15 & 77465 & 882001 \\
2 & 1.21 & 0.13 & 81541 & 870194 \\
2.2 & 1.09 & 0.12 & 89696 & $\mathbf{8 6 0 5 3 2}$ \\
2.25 & 1.07 & 0.12 & $\mathbf{9 1 7 3 5}$ & 860200 \\
\hline
\end{tabular}

tions for the cases where $p_{b}$ and $p_{d}$ are fixed to zero, respectively. Table 2 shows solutions of the optimization for varying $\gamma$.

We tried to extend these experiments to the case of several ticket types, but found out that it is hard to construct demand functions for this case by hand. In Section 6 we investigate an approach for obtaining such demands.

Discussion. A very rough summary of the results is that the fares should double while the demand halves. As noted above, the consequences that can be drawn from these results are limited. Nevertheless, this result can be interpreted as follows: When maximizing revenue it pays off to exploit the passengers which depend on public transport as much as possible. In reality, often such a plan is politically and socially not possible. However, it might explain why many public transport companies try to increase the fares as much and as often as possible. On the other hand, the fares in public transport cannot be seen independent from private transport. (The model in Section 6 tries to incorporate both modes of transport). It may very well be that such a result also holds for private transport, i.e., taxes on cars and fuel should double while the demand halves, hence maximizing the revenue of the tax collector.

\section{A Discrete Choice Model}

In this section we investigate the behavior of passengers when several discrete alternatives of travel are present. The decisions of passengers depend on the utilities (i.e., a combination of fares, travel time, comfort, etc.) of the corresponding alternatives. Assuming that the alternative with highest utility is chosen, this leads to a discrete choice model. Considering the fare as variable and the other parameters as fixed, this gives a demand function 
which can be used in our model for FPP. This demand function is more realistic than the previous example.

First we introduce discrete choice models and, in particular, logit models, which are well known in the literature, see for instance Ben-Akiva and Lerman [2]. Then we apply and extend the model to the fare planing problem and discuss a concrete example. The additional feature is the incorporation of the number of trips in a given time horizon, which we assume to be random.

\subsection{A General Discrete Choice Model}

In the context of public transport, each passenger chooses among a set $A=\left\{a_{1}, \ldots, a_{n}\right\}$ of alternatives for the travel mode, e.g., single ticket, monthly ticket, bike, car travel, etc. Associated with each alternative $a \in A$ is a certain utility $U_{a}$ which may depend on the passenger. For such a discrete choice model, it is assumed that the utilities cannot be completely be estimated. Therefore each utility decomposes into an observable part, the deterministic utility $V_{a}$, and a random utility, the disturbance term $\nu_{a}$ :

$$
U_{a}=V_{a}+\nu_{a} .
$$

Assuming that each passenger chooses the alternative with the highest utility, the probability of choosing alternative $a_{i}$ is

$$
P_{a_{i}}:=\mathbb{P}\left[V_{a_{i}}+\nu_{a_{i}} \geq \max _{j \in\{1, \ldots, n\} \backslash\{i\}}\left(V_{a_{j}}+\nu_{a_{j}}\right)\right] .
$$

In general, it is impossible to find an analytical expression for this probability. The following logit model is a discrete choice model with specific assumptions on the distribution of each $\nu_{a}$ which make the analysis possible.

\subsection{Logit Model}

In the logit model the disturbance terms $\nu_{a}$ are assumed to be independent identically Gumbel distributed. This distribution is defined as

$$
F(c)=\exp \left(-e^{-\mu(c-\eta)}\right) .
$$

The density function of the Gumbel distribution is defined as

$$
f(c)=\mu e^{-\mu(c-\eta)} \exp \left(-e^{-\mu(c-\eta)}\right),
$$

where $\eta$ is a location parameter and $\mu>0$ is a scale parameter. For convenience, we assume $\eta=0$ in the following.

Consider the probability for alternative $a_{i}$ as defined in (4). It turns out that utility $U_{a_{j}}=V_{a_{j}}+\nu_{a_{j}}$ for each alternative $a_{j}$ is also Gumbel distributed with parameters $\left(V_{a_{j}}, \mu\right)$. A characteristic of independent Gumbel 
distributed random variables with identical scale parameter $\mu$ is that their maximum is Gumbel distributed, too. More precisely, the maximum in (4) is a Gumbel distributed random variable $\tilde{\nu}$ with parameters $(\tilde{V}, \mu)$, where

$$
\tilde{V}=\frac{1}{\mu} \ln \sum_{\substack{j=1 \\ j \neq i}}^{n} e^{\mu V_{a_{j}}}
$$

We obtain for the probability for choosing alternative $a_{i}$ :

$$
P_{a_{i}}=\mathbb{P}\left[V_{a_{i}}+\nu_{a_{i}} \geq \tilde{\nu}\right]=\mathbb{P}\left[V_{a_{i}} \geq \tilde{\nu}-\nu_{a_{i}}\right] .
$$

The difference of two independent Gumbel distributed random variables is logistically distributed; in this case, $\tilde{\nu}-\nu_{a_{i}}$ in (5) is logistically distributed with distribution

$$
\tilde{F}(x)=\frac{1}{1+e^{\mu(\tilde{V}-x)}} .
$$

Hence for the probability for alternative $a_{i}$ we obtain

$$
P_{a_{i}}=\frac{1}{1+e^{\mu\left(\tilde{V}-V_{a_{i}}\right)}}=\frac{e^{\mu V_{a_{i}}}}{\sum_{j=1}^{n} e^{\mu V_{a_{j}}}} .
$$

\subsection{Example with Three Alternatives}

In this section we apply a logit model to the fare planning problem and optimize over the fares of public transport in the model. For concreteness, we discuss our approach on an example with three alternatives and two variables, but the approach can easily be generalized. The choice among the alternatives will depend on the number of trips during a given time horizon, which we assume to be random. The goal is then to maximize the expected revenue.

The three alternatives in our example are: $a_{1}=$ "reduced ticket", $a_{2}=$ "standard ticket", and $a_{3}=$ "car", for each pair $(s, t)$ of nodes. Our model works within a given time horizon $T$, for instance one month. Each passenger is allowed to choose one of these three modes of travel for all trips during the time horizon. Hence, we assume that passengers do not mix the alternatives in the time horizon and that it is not possible to skip trips.

The passenger has to pay a distance dependent fare with the standard ticket for each trip. For reduced tickets this fare is reduced, but the passenger has to pay a basic fare once per time horizon. For alternative "car", the passenger has to pay a fixed price and distance dependent operating costs. Below, the fares of the public transport alternatives $a_{1}$ and $a_{2}$ will be considered variable, while the other parameters will be fixed. 
We work with a nonnegative random variable $X_{s t}$ indicating the number of trips of a single passenger between $s$ and $t$ during $T$. Usually $X_{s t}$ is a discrete random variable, but our model allows for continuous probability as well. In practice, one tries to estimate the number $n_{s t}^{i}$ of passengers that make a given number of trips $i$ between $s$ and $t$ during $T$. From this one gets a discrete probability distribution by setting

$$
\mathbb{P}\left[X_{s t}=i\right]:=\frac{n_{s t}^{i}}{\sum_{j} n_{s t}^{j}} .
$$

For each OD-pair $(s, t) \in D$ we define the following: Let $U_{s t}^{a}$ be the utility for each alternative $a$. Let $p_{B}$ be the basic fare during $T$ and $p_{s t}^{a_{1}}$ the fare of one trip for alternative "reduced ticket". Let $p_{s t}^{a_{2}}$ be the fare of one trip with alternative "standard ticket", where we assume that $p_{s t}^{a_{2}}>p_{s t}^{a_{1}}$. Let $P^{a_{3}}$ the fixed costs for a car in the time horizon and $p_{s t}^{a_{3}}$ the operating costs for a car. Note that the distance between $s$ and $t$ is already accounted for in $p_{s t}^{a_{1}}$, $p_{s t}^{a_{2}}$, and $p_{s t}^{a_{3}}$.

In this example, we let the deterministic utility $V_{s t}$ include the fare and the travel time $t_{s t}^{a_{i}}$ for one trip of the corresponding alternative. The two parts are weighted by parameters $\delta_{1}, \delta_{2}>0$. The utilities (with respect to $T$ and $(s, t) \in D)$ for the alternatives are:

$$
\begin{array}{lr}
U_{s t}^{a_{1}}=-\delta_{1}\left(p_{B}+p_{s t}^{a_{1}} \cdot X_{s t}\right)-\delta_{2} \cdot t_{s t}^{a_{1}} \cdot X_{s t}+\nu_{s t}^{a_{1}} & \text { "reduced ticket" } \\
U_{s t}^{a_{2}}=-\delta_{1} \cdot p_{s t}^{a_{2}} \cdot X_{s t}-\delta_{2} \cdot t_{s t}^{a_{2}} \cdot X_{s t}+\nu_{s t}^{a_{2}} & \text { "standard ticket" } \\
U_{s t}^{a_{3}}=-\delta_{1}\left(P^{a_{3}}+p_{s t}^{a_{3}} \cdot X_{s t}\right)-\delta_{2} \cdot t_{s t}^{a_{3}} \cdot X_{s t}+\nu_{s t}^{a_{3}} & \text { "car". }
\end{array}
$$

Note that in this model all travelers for an OD-pair $(s, t) \in D$ are treated the same, i.e., they all have the same alternatives and parameters.

We now can derive formulas for the probabilities of choice. The probability for a traveler from $s$ to $t$ to choose alternative $a$ with respect to the number of trips $X_{s t}$ can easily be computed using (6). For instance, for alternative $a_{1}$ ("reduced ticket") we obtain the conditional probability:

$$
P_{s t}^{a_{1}}(k):=\mathbb{P}\left[U_{s t}^{a_{1}} \geq \max \left\{U_{s t}^{a_{2}}, U_{s t}^{a_{3}}\right\} \mid X_{s t}=k\right]=\frac{e^{\mu V_{s t}^{a_{1}}}}{e^{\mu V_{s t}^{a_{1}}}+e^{\mu V_{s t}^{a_{2}}}+e^{\mu V_{s t}^{a_{3}}}} .
$$

The expected number of passengers using a reduced ticket then is:

$$
E_{s t}^{a_{1}}:=d_{s t} \sum_{k=0}^{\infty} P_{s t}^{a_{1}}(k) \mathbb{P}\left[X_{s t}=k\right]
$$

where $d_{s t}$ is the number of persons that have to travel between $s$ and $t$, $(s, t) \in D$. The other numbers can be computed similarly. We obtain the 
expected total revenue (over all pairs $(s, t) \in D$ ) for public transport:

$$
\begin{aligned}
& r\left(p_{B}, p_{s t}^{a_{1}}, p_{s t}^{a_{2}}\right)= \\
& \quad \sum_{(s, t) \in D} d_{s t} \sum_{k=0}^{\infty}\left(P_{s t}^{a_{1}}(k) \cdot\left(p_{B}+p_{s t}^{a_{1}} \cdot k\right)+P_{s t}^{a_{2}}(k) \cdot p_{s t}^{a_{2}} \cdot k\right) \mathbb{P}\left[X_{s t}=k\right] .
\end{aligned}
$$

Note that this total expected revenue is differentiable.

If no disturbance terms would be present, all passengers for a given ODpair $(s, t) \in D$ with the same number of trips $X_{s t}$ would choose the same alternative. Furthermore, in this case the revenue can be discontinuous since there is a certain fare for which all travelers change their decision from the public transport alternatives to car travel and are then not accounted for in (7). Hence, the introduction of the disturbance terms, i.e., the logit model part, smoothes the discrete choice model.

\subsection{Computational Results}

We are now ready to perform computational experiments with the model described in the previous section.

For our computational experiments we again worked on the data for the Netherlands. Thus, we are given an OD-matrix which captures the number of passengers using the intercity network. We assume that this constitutes the only source of traffic in public transport. We estimated the total number of travelers (including car travel) by using our model as follows. Given the current fares and traveling times, we calculated the percentage of passengers using public transport with our model and then computed the number of total travelers from it. This should give an (extremely) rough estimation of the real number of travelers.

Additionally, we obtained data about the current fares, (railway) distances, and traveling time from the web site [10]. The distances and traveling times for using a car were obtained from the routing planer [9]; here we entered the corresponding train stations as start and end points of the travel.

Our time horizon is one month. The number of trips $X_{s t}$ were chosen as discrete identically independent random variables with the probabilities given in Figure 3 for every OD-pair $(s, t)$; the probabilities are centered around 30 trips and are nonzero between 0 and 60 .

We assumed that $p_{s t}^{a_{2}}=2 \cdot p_{s t}^{a_{1}}$, i.e., for a reduced ticket one has to pay half of the distance dependent fare of the standard ticket. Furthermore, we let $p_{s t}^{a_{1}}=p_{d} \cdot \ell_{s t}^{P}$, where $\ell_{s t}^{P}$ is the distance in kilometers between $s$ and $t$ for the public transport and $p_{d}$ is a variable to be determined. The other variable is the basic fare $p_{B}$ for reduced tickets, paid once per month. The travel time $t_{s t}^{a_{1}}=t_{s t}^{a_{2}}$ for the public transport alternatives is measured in minutes, as obtained from [10]. 

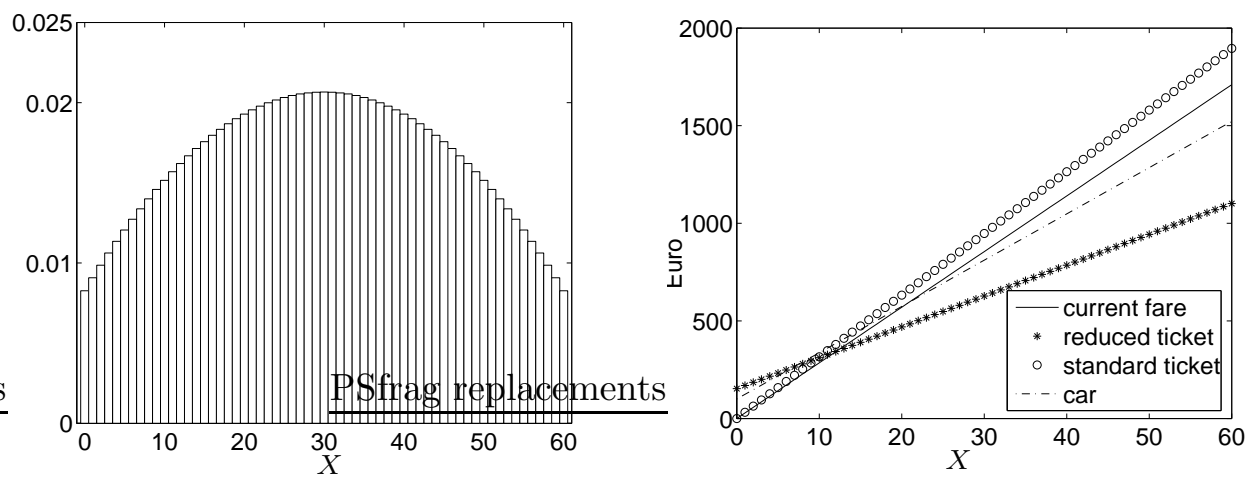

Figure 3: Left: The discrete random variable $X$ for the number of trips. Right: Example of fares in dependence on the number of trips for a selected OD-pair $(s, t)$ of high distance.
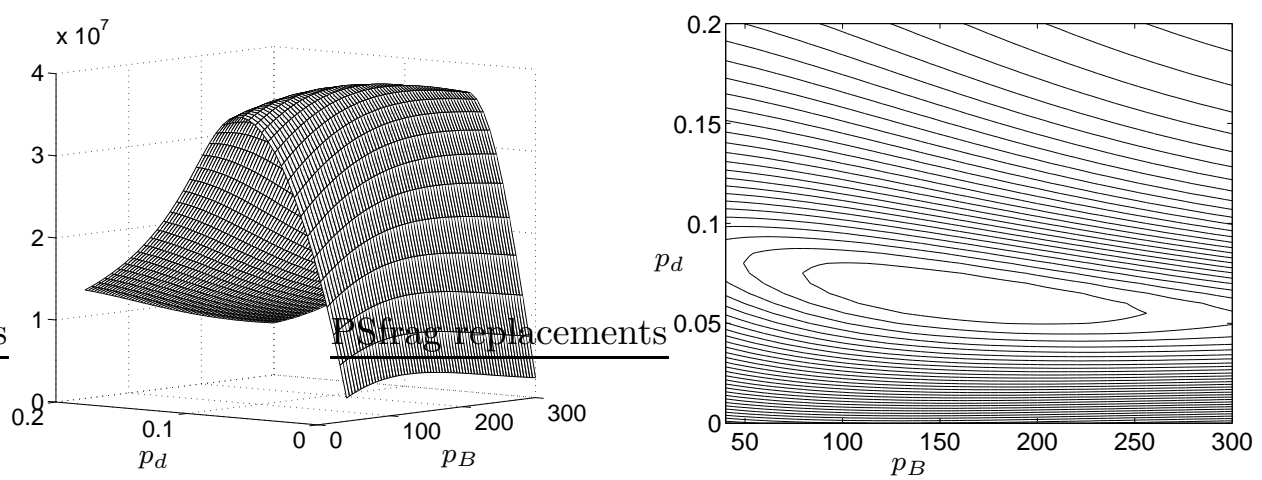

Figure 4: Discrete choice model. Left: Total revenue. The optimum is at $p_{B} \approx 153.30$ and $p_{d} \approx 0.06$. Right: A contour plot of the total revenue.

Table 3: Computed fares and currently valid fares for selected trips. The current fares are $p^{0}$, the current demand is $d^{0}$. For each alternative $a, E_{s t}^{a}$ gives the expected number of passengers traveling with $a$ from $s$ to $t$. For alternative "reduced ticket", $F_{s t}^{a_{1}}=p_{B}+$ $p_{d} \cdot \ell_{s t}^{P} \cdot 30$ is its expected fare, and $F_{s t}^{a_{2}}=2 \cdot p_{d} \cdot \ell_{s t}^{P} \cdot 30$ is the expected fare for alternative "standard ticket". $\ell$ denotes the distance between the selected endpoints (with public transport) of the travel.

\begin{tabular}{rrrrlrrr}
\hline$\ell$ & $d^{0}$ & $p^{0} \cdot 30$ & \multicolumn{1}{c}{$E_{s t}^{a_{1}}$} & $F_{s t}^{a_{1}}$ & \multicolumn{1}{c}{$E_{s t}^{a_{2}}$} & \multicolumn{1}{c}{$F_{s t}^{a_{2}}$} & \multicolumn{1}{c}{$E_{s t}^{a_{3}}$} \\
\hline 11 & 1456 & 63 & 345.0 & 174.16 & 1289.3 & 41.71 & 445.5 \\
35 & 5826 & 156 & 2211.8 & 219.66 & 5362.8 & 132.72 & 4756.7 \\
50 & 1829 & 213 & 801.4 & 248.10 & 1359.9 & 189.60 & 475.7 \\
106 & 720 & 420 & 649.5 & 354.28 & 417.1 & 401.95 & 257.2 \\
150 & 24 & 576 & 38.5 & 437.70 & 14.8 & 568.80 & 22.4 \\
202 & 77 & 723 & 38.3 & 536.30 & 63.1 & 765.98 & 1303.6 \\
250 & 1 & 855 & 2.6 & 627.30 & 0.5 & 948.00 & 2.3 \\
303 & 14 & 999 & 25.5 & 727.79 & 2.8 & 1149.00 & 3.5 \\
\hline
\end{tabular}


We set the fixed costs $P^{a_{3}}$ of car travel to be $100 €$, as an estimation for insurance and car repair for one month. The operating costs of car travel were set to $p_{s t}^{a_{3}}=0.1 \cdot \ell_{s t}^{C}$, where $\ell_{s t}^{C}$ is the distance in kilometers between $s$ and $t$ for the quickest route obtained with the routing planer [9]; hence, we estimated the operating cost for one kilometer to be $0.1 €$. The travel time for a car $t_{s t}^{a_{3}}$ is measured in minutes for the quickest route with the routing planer [9].

The random utilities in the logit part were chosen to be Gumbel distributed with parameters $\mu=1, \eta=0$. In the utility functions we used the scaling parameters $\delta_{1}=1$ and $\delta_{2}=0.1$, i.e, 10 minutes of travel time is worth one Euro.

On the right of Figure 3, one sees the fares for the different alternatives and the current fare in dependence on the number of trips for a particular $(s, t) \in D$ with high distance.

The resulting expected revenue function in dependence on the basic fare $p_{B}$ and the distance fare $p_{d}$ for reduced tickets is shown in Figure 4. The optimal fare is determined by $p_{B} \approx 153.30$ and $p_{d} \approx 0.06$. For the computations again we used standard methods, in this case a Newton-type method in Matlab 7 for finding the critical point (the result was confirmed by the Nelder-Mead method).

Table 3 illustrates the results for selected OD-pairs. The third column gives the current fares for public transport (here only a "standard ticket" is available). The second column lists the corresponding number of passengers - these numbers arise from the original OD-matrix. Then for each alternative $a$, we list the corresponding expected number of passengers $E_{s t}^{a}$ traveling between the selected pair $(s, t)$. Since the expectation of $X_{s t}$ is 30, $F_{s t}^{a_{1}}=p_{B}+p_{d} \cdot \ell_{s t}^{P} \cdot 30$ is the expected fare for alternative "reduced ticket", and $F_{s t}^{a_{2}}=2 \cdot p_{d} \cdot \ell_{s t}^{P} \cdot 30$ is the expected fare for alternative "standard ticket".

The data show that with increasing distance, alternative "reduced ticket" becomes more attractive than alternative "standard ticket" since the contribution of the basic fare becomes smaller and the reduction of the distance dependent fare becomes more important. Furthermore, in each case the demand for public transport with optimal fares is always higher than the demand with the current fares. This is in contrast to the example in Section 5. An explanation may be that one cannot exploit passengers as in this first example, because at a certain point they switch to alternative car.

We also performed experiments with the situation where the public transport alternatives are "single ticket" and "monthly ticket"; this also yields a model with three alternatives and two variables. We opted for the presentation of the above example since monthly tickets are somewhat uncommon in rail transport. 


\section{References}

[1] H. J. BAUm, Free public transport, Journal of Transport Economics and Policy, Vol. 7, pp. 3-19, 1973.

[2] M. Ben-Akiva and S. R. Lerman, Discrete Choice Analysis: Theory and Application to Travel Demand, MIT-Press, Cambridge, 1985.

[3] M. Bussieck, Gams model lop.gms: Line optimization. http://www.gams. com/modlib/libhtml/lop.htm.

[4] M. R. Bussieck, T. Lindner, And M. E. LüBbecke, A fast algorithm for near optimal line plans, Math. Methods Oper. Res., Vol. 59, No. 2, pp. 205220, 2004.

[5] P. Cerwenka, Glanz und Elend der Elastizität, Der Nahverkehr, Vol. 6, pp. 28-33, 2002.

[6] J. F. Curtin, Effect of fares on transit riding, Highway Research Record 213, Washington D.C., 1968.

[7] H. W. Hamacher And A. SchöBel, On fair zone designs in public transportation, in Proc. of the Sixth International Workshop on Computer-Aided Scheduling of Public Transport (CASPT), Lisbon, Portugal, 1993, J. R. Daduna, I. Branco, and J. M. P. Paixão, eds., Vol. 430 of Lecture Notes in Economics and Mathematical Systems, Springer-Verlag, Berlin, Heidelberg, 1995, pp. 8-22.

[8] — Design of zone tariff systems in public transportation, Tech. Report 21, Frauenhofer Institut für Techno- und Wirtschaftsmathematik, 2001.

[9] Routing planer. http://www.de.map24.com.

[10] Reisadvies. http://www.9292ov.nl.

[11] P. A. Samuelson, Economics, MacGraw-Hill, New York, 12th ed., 1986.

[12] K. Storchmann, Externalities by automobiles and fare-free transit in germany - a paradigm shift, Journal of Public Transportation, Vol. 6, No. 4, pp. 89-105, 2003.

[13] M. Vrtic and K. W. Axhausen, Modelle der Verkehrsmittelwahl aus RPund SP-Datengrundlage, in Heureka, Tagungsband, Karlsruhe, pp. 293-309, 2002, Forschungsgesellschaft für Straßen- und Verkehrswesen.

[14] K. Walther, Der Preiselastizitätsfaktor im ÖPNV und seine Bestimmungsgrößen, Der Nahverkehr, Vol. 1-2, pp. 33-36, 1993. 